\title{
ESTUDIO EXPERIMENTAL DEL EFECTO HIDROLÓGICO DE CUBIERTAS VERDES PARA SU UTILIZACIÓN EN EL ÁREA METROPOLITANA DEL GRAN RESISTENCIA
}

\author{
NéstorHolsbach,Jorge Pilar ${ }^{(*)}$,Claudia Pilar \\ Departamento de Hidráulica, Facultad de Ingeniería, Universidad Nacional del Nordeste.Resistencia, Chaco, \\ Argentina. \\ ${ }^{(*)}$ e-mail: jvpilar@gmail.com
}

\begin{abstract}
RESUMEN
Se presentan los resultados preliminares de un estudio sobre modelo físico, referidos a la eficacia y eficiencia hidrológicas de cubiertas verdes para su utilización en el Área Metropolitana del Gran Resistencia (AMGR), Chaco.Las cubiertas verdes de tipo extensivas son sistemas que se colocan sobre techos tradicionales para disminuir los efectos de la impermeabilización del suelo urbano, buscando laminar los hidrogramas de escurrimiento que se generan. Están compuestas por varias capas construidas con distintos elementos, con características y propiedades diferentes, estando la capa superior constituida por un sustrato orgánico, de poco espesor, que permite el crecimiento de vegetación de bajo porte y por el que recibe el nombre de cubiertas verdes. El aumento de la impermeabilización asociada a la creciente urbanización, la baja pendiente natural del terreno y su localización en una zona de riesgo hídrico hacen propicia la implementación de este sistema en las ciudades que conforman el Área Metropolitana del Gran Resistencia como una medida de mitigación que permitiría minimizar los efectos de precipitaciones de elevada intensidad, reduciendo y retardando el agua de escurrimiento directo, colaborando a evitar (o por lo menos minimizar) los anegamientos provocados por las lluvias.En el presente trabajo se buscó evaluar cualitativa y cuantitativamente el efecto de este tipo de techos. Para ello, se construyó un modelo físico de la cubierta extensiva, utilizándose además otra de tipo plana tradicional como contraste. Los resultados preliminares obtenidos mostraron un notorio efecto amortiguador y retardador del escurrimiento de este tipo de cubiertas.
\end{abstract}

Palabras Clave: Techos verdes, drenaje pluvial urbano, hidrología urbana.

\begin{abstract}
Preliminary results of a study on a physical modeling referred to hydrologic effectiveness and efficiency are presented herefor use in the Greater Resistencia Metropolitan Area (AMGR), Chaco.Extensive green roofs are systems that are placed on traditional roofs to reduce the effects of waterproofing of urban land in order to laminate runoff hydrograms they generate. They are composed of several layers built with different elements, having different characteristics and properties, the upper layer consisting of an organic substrate, and thin thickness, which allows growth of low size vegetation and for what are known green roofs.Increasing on waterproofing associated to urbanization, the low natural slope of the land and its location in an area of water risk makes it conducive implementing this system in the cities making up Great Resistencia Metropolitan Area, as a mitigation measure that would minimize effects of high intensity rainfall, thus reducing and delaying direct runoff water, helping to avoid (or at least minimize) flooding caused by rainfall.The aim of this work was evaluate qualitatively and quantitatively effects of this type of roofing. To do this, a physical model of the extensive roof was constructed, by using a traditional flat roof as a comparison.Preliminary results obtained showed an important damping and retarding effect of the runoff of this type of roof.
\end{abstract}

Key words:Green roofs, urban storm drainage, urban hydrology. 


\section{INTRODUCCIÓN}

Desde un punto de vista hidrológico, el crecimiento de la urbanización se ve reflejado en el incremento de áreas impermeables, lo que provoca un mayor volumen de escurrimiento superficial, un aumento del caudal de pico de ese escurrimiento, además de una aceleración de ese proceso.

Ello tiene un efecto significativo en muchas de las grandes ciudades de la región NEA, especialmente las que se localizan en áreas planas, con drenaje complejo, que ante la ocurrencia de precipitaciones intensas (inclusive de algunas de mediana intensidad) quedan paralizadas por los anegamientos producidos. Y esto se torna preocupante en el contexto de cambio climático por el que se está transitando.

Como respuesta a esta problemática, algunas ciudades, siguiendo la tendencia internacional liderada por Francia y ciudades como Copenhague o Barcelona, dictaron normativas que promueven el uso de los denominados techos verdes.

Tal es el caso de Buenos Aires, que promulgó en 2012 la Ley 4428 de Techos y Terrazas Verdes (Legislatura de la Ciudad Autónoma de Buenos Aires, 2012); esta iniciativa fue incluida posteriormente en el Código de Edificación de la Ciudad (CE). También la ciudad de Córdoba promulgó una normativa similar en el año 2016, cuyos términos originales fueron flexibilizados por la oposición de los promotores inmobiliarios.

Sin embargo, en la Región NEA aún no se han puesto en vigencia normativas similares, a pesar que ciudades como Resistencia han sido pioneras en el uso obligatorio de medidas no estructurales y de mitigación como parte del abordaje de la problemática del drenaje pluvial urbano (Depettris y Pilar, 2001).

El Área Metropolitana del Gran Resistencia (AMGR) se localiza sobre la Llanura ChacoPampeana, sobre la margen derecha del río Paraná, a un par de decenas de kilómetros de la desembocadura del río Paraguay, en una zona de alto riesgo de inundaciones fluviales. Muestra una urbanización que crece a una tasa del orden de 2 hectáreas por semana (Scornik, 1998), no siempre planificada adecuadamente, lo que sumado a la baja energía del relieve (pendiente regional inferior a $10 \mathrm{~cm} / \mathrm{km}$ ) son condiciones de contexto que hacen propicia la implementación de los techos verdes, que en ese escenario complejo podrían servir como gran ayuda en la búsqueda de un sistema de drenaje pluvial urbano eficaz y eficiente.

El aumento de la impermeabilización asociada a la creciente urbanización provoca una reducción de la infiltración, de la percolación, del escurrimiento subsuperficial y del subterráneo, incrementando el volumen de escurrimiento superficial.

Entonces, el uso generalizado de los techos verdes permitiría minimizar los efectos de precipitaciones de elevada y mediana intensidad, reduciendo y retardando el agua de escurrimiento directo, mitigando los anegamientos por lluvias de calles, veredas y lotes urbanos (Depettrisy Pilar, 2001).

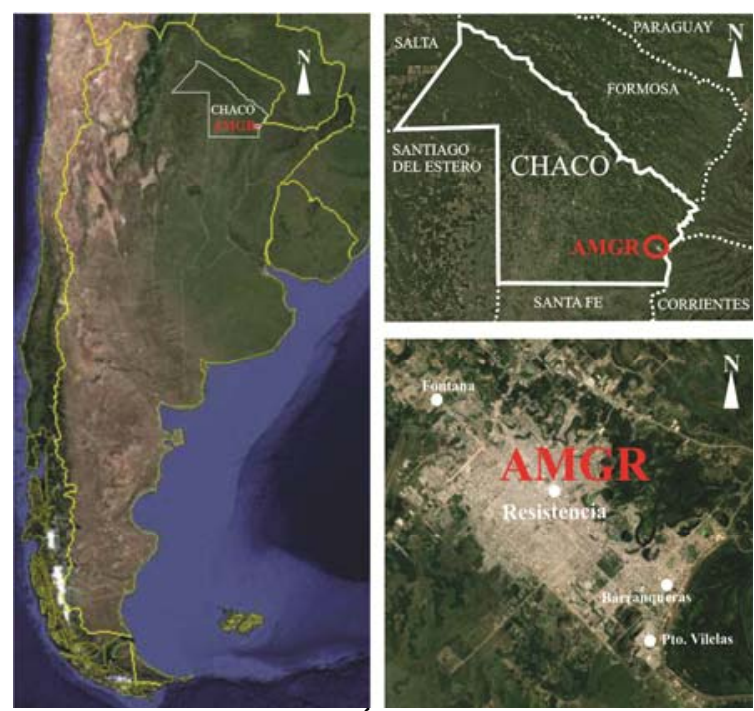

Figura1. Localización del Área Metropolitana del Gran Resistencia (Fuente: imágenes de Google Earth intervenidasgráficamente por los autores).

La construcción de cubiertas verdes permitiría retardar el flujo del agua de los techos hacia la red colectora pluvial, como consecuencia de la absorción radicular y delefecto almacenador del manto de tierra.

Por lo expuesto, queda claro que los techos verdes constituyen una estrategia constructiva alternativa que redundará en beneficios ambientales, paisajísticos y también técnicos.

Si bien este tipo de cubiertas es mencionado en numerosas publicaciones, no es fácil encontrar datos cuantitativos de sus efectos desde una óptica hidrológica.

Un esquema de funcionamiento sería como el mostrado en la Figura 2. 


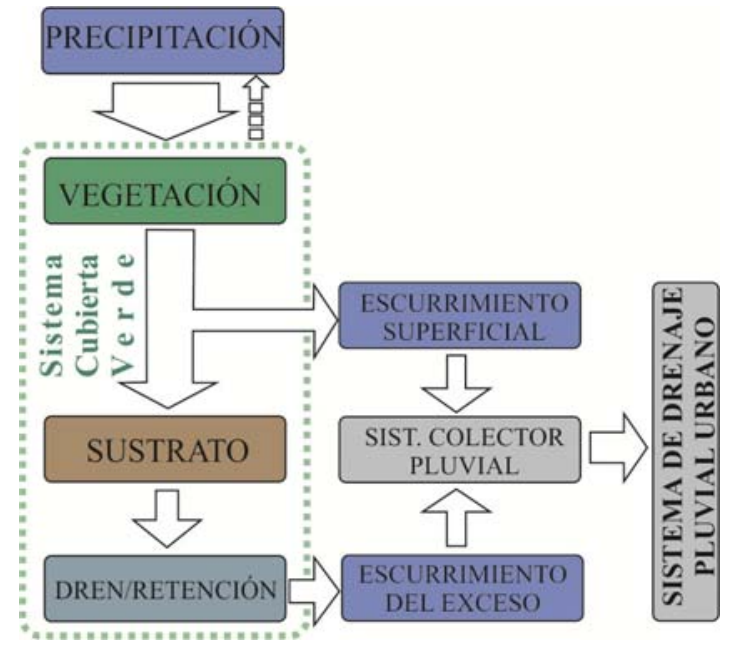

Figura2. Esquema de funcionamiento hidrológico de una cubierta tipo extensiva (Fuente: elaboración propia).

La norma IRAM No 11.649 define al techo como: “el sistema de uno o más elementos que cierra o que cubre la parte superior de un edificio, contribuyendo a crear una determinada situación de uso y manteniéndolo frente a una determinada situación ambiental”. Por lo tanto, es una de las partes del edificio, al igual que las paredes, que suministran protección contra los agentes externos (Chandíasy Ramos, 2009). Este sistema está compuesto principalmente por dos elementos fundamentales: la cubierta, constituida por materiales resistentes a las variaciones térmicas e hidráulicas, y la estructura, que le brinda estabilidad para soportar los esfuerzos externos.

Las cubiertas verdes tipo extensivas (o ecológicas) son sistemas para construir techos, compuestas por varias capas elaboradas con diferentes elementos, con características y propiedades distintas. Según Britto Correa (2001) la configuración básica de las capas es la siguiente:

- Vegetación: deben ser especies que se adapten a las condicionantes impuestas por el clima, de baja profundidad radicular, tapizantes, autorregenerativas; las recomendadas son las del género Sedum (suculentas) y Gramíneas.

- Sustrato vegetal o inorgánico: capaz de brindar nutrientes a la vegetación a implantar y retener agua en sus poros. El espesor de la capa varía entre 8 a $10 \mathrm{~cm}$, aproximadamente.

- Filtro geotextil: membrana que impide el paso de los granos finos del sustrato y permite el drenaje del agua.

- Capa de drenaje: manto que extrae el exceso de agua del sustrato.

- Aislamiento térmico: aplicado para atender las necesidades térmicas del edificio.

- Protección contra raíces: evita el ingreso de las raíces al sistema estructural del techo.

- Lámina de impermeabilización: protege al edificio del ingreso de agua a través del techo.

- Estructura resistente: sostén de la cubierta dimensionada para soportar el peso de las capas superiores y eventuales sobrecargas.

En la Figura 3 se puede observar una sección típica de una cubierta ajardinada, en donde se representa cada componente de la misma.

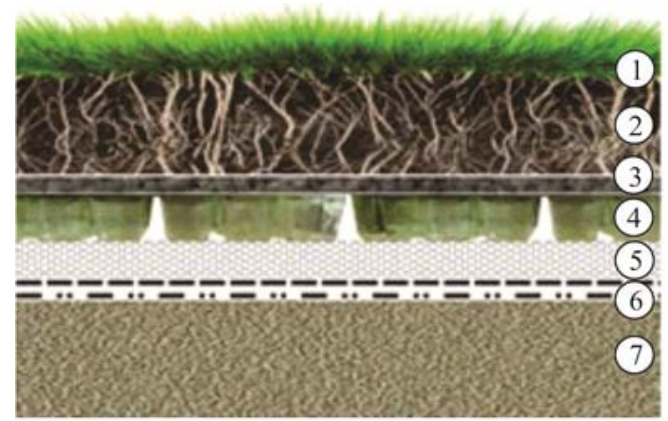

Referencias: 1. Vegetación; 2. Sustrato; 3. Capa filtrante; 4. Capa drenante; 5. Aislamiento Térmico; 6. Barrera antiraíces y barrera hidráulica y 7 . Estructura portante.

Figura 3. Componentes de una cubierta verde tipo extensiva.

Este tipo de superficies presenta ventajas que benefician al entorno urbano $\mathrm{y}$ a la obra arquitectónica (Pilar et al., 2009). Algunos de los beneficios más relevantes según Machado et al. (2000) son:

- Retención de polvo y sustancias contaminantes propias de las ciudades.

- Incremento dela superficie verde en ciudades.

- Protección contra la radiación solar en edificios.

- Aumento de la eficacia térmica de la cubierta.

- Absorción del ruido.

- Reducción del efecto de la "isla de calor".

- Reducción de la carga de agua en las canalizaciones, de los costos de depuración de aguas residuales y minimización de los riesgos de inundación en las ciudades.

Los objetivos del presente trabajo fueron analizar los efectos de la incorporación de vegetación en techos para disminuir el impacto ambiental de las superficies construidas en los entornos urbanos, minimizando las consecuencias hidrológicas de la 
impermeabilización creciente en las ciudades. Para ello, se buscó evaluar cualitativa y cuantitativamente los efectos de este tipo de cubiertas en caso de ser empleadas en el AMGR.

Se recurrió a un modelo físico de la cubierta extensiva elaborado al efecto, construido con materiales alternativos, pero con características similares a los ofrecidos en el mercado, dado que aún no se encuentran disponibles en la región materiales específicos o que resultan muy onerosos, por la baja o nula utilización de este tipo de cubiertas. Los resultados preliminares obtenidos mostraron un interesante efecto retardador de escurrimiento de este tipo de cubiertas.

\section{MATERIALES Y MÉTODOS}

Se utilizó un modelo físico que representa una porción de la cubierta extensiva, de $30 \mathrm{~cm}$ de ancho por $40 \mathrm{~cm}$ de largo y $15.5 \mathrm{~cm}$ de espesor. El recipiente de contención fue de PVC transparente, de 42 litros de capacidad, en el cual se realizaron perforaciones en un extremo para permitir el drenaje. Se le dio una pendiente de fondo de 2 $\mathrm{cm} / \mathrm{m}$, semejante a las pendientes utilizadas en las cubiertas planas tradicionales (losas), y se utilizó una de estas cubiertas planas como contraste.

El modelo se ejecutó con los siguientes materiales: goma espuma en lámina, de $5 \mathrm{~mm}$ de espesor, que cumple con la función de filtrar el agua evitando la erosión del sustrato; celdas de cultivo de polietileno reciclado, de $45 \mathrm{~mm}$ de alto, cuya finalidad fue retener una parte del agua infiltrada y drenar el resto; sustrato orgánico de la región (tierra negra), de 100 mm de espesor. Para la vegetación se optó por el uso de césped (Figura 4).

Los dos primeros elementos constituyentes son de características similares a los ofrecidos en el mercado (aunque no en el comercio local), dado que aún no se encuentran disponibles en la región materiales específicos, por la baja (casi nula) utilización de este tipo de cubiertas.

Para la construcción del modelo físico se siguieron los siguientes pasos:

1) Se perforó del recipiente contenedor en su cara menor (la de $30 \mathrm{~cm}$ de longitud).

2) Se colocó la lámina de espuma de poliuretano (goma espuma) sobre el fondo del recipiente.
3) Se ubicó el sistema de drenaje-retención sobre la capa anterior.

4) Nuevamente se colocó otra capa de goma espuma.

5) Se distribuyó el sustrato (tierra negra vegetal) de forma suelta, esparciéndola manualmente en capas de $2 \mathrm{~cm}$ aproximadamente hasta lograr el espesor de $8 \mathrm{~cm}$.

6) Se sembraron 6 gramos de semillas de césped (50 gramos $/ \mathrm{m}^{2}$ ).

7) Finalmente, se agregaron otros $2 \mathrm{~cm}$ de suelo vegetal, para completar los $10 \mathrm{~cm}$ de capa del sustrato.

En la Figura 4 se muestra el modelo físico utilizado.

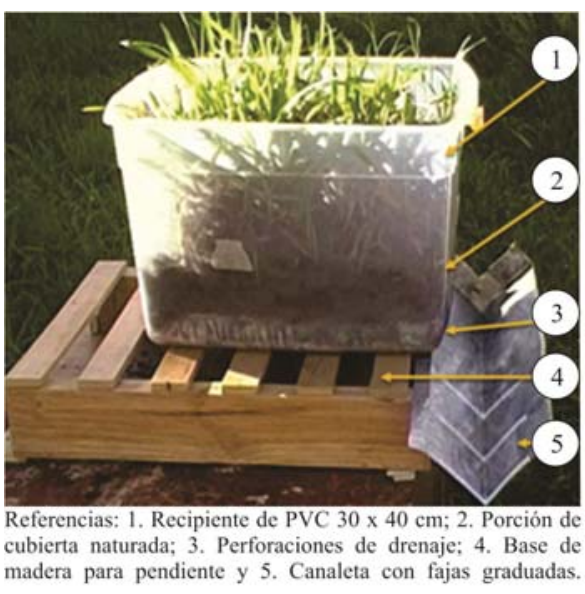

Figura 4.Modelo físico utilizado.

Se realizaron 5 ensayos consecutivos, de 2 minutos de duración cada uno. En todos ellos se simuló una precipitación de 4 litros, sobre la superficie de 0.123 $\mathrm{m}^{2}$ y se midieron los caudales drenados. En un recipiente se captó el agua filtrada en cada experiencia (Figura 5).

La misma operación se realizó sobre una cubierta plana impermeable (losa), para la cual se registró el hidrograma de escurrimiento directo. Posteriormente, se compararon ambos hidrogramas y se analizaron sus parámetros característicos: tiempo de base, caudal pico y el tiempo en el que éste se produce.

\section{RESULTADOS OBTENIDOS}

Los resultados obtenidos de la simulación en modelo físico se pueden apreciar en la Figura 6.

Se puede notar una diferencia marcada en los hidrogramas de escurrimiento de la losa y de la cubierta verde: 
- Cubierta de losa plana: tiempo base 119 segundos; tiempo al pico 11 segundos; con respecto al caudal pico el mismo es de $81.8 \mathrm{~cm}^{3} / \mathrm{s}$.

- Cubierta verde: tiempo base 480 segundos (incremento del 400\%); tiempo al pico 30 segundos (incremento de 273\%); caudal pico 17 $\mathrm{cm}^{3} / \mathrm{s}$ (reducción de $79.2 \%$ ).

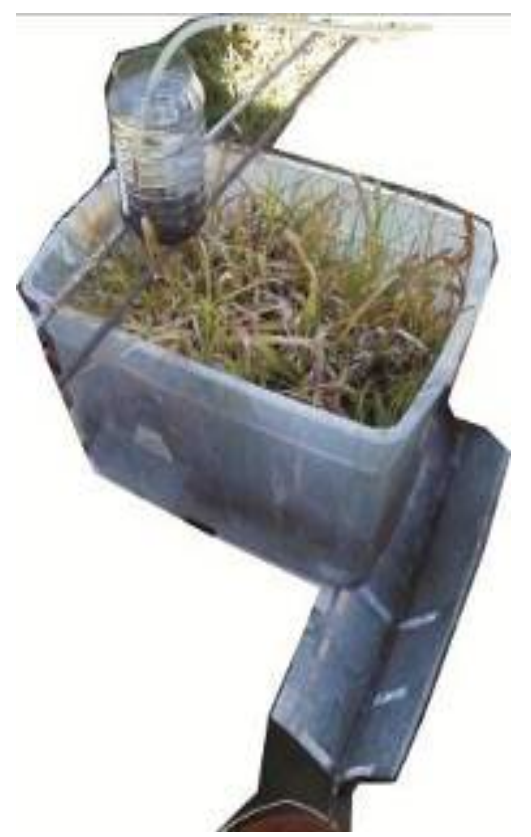

Figura 5.Ensayo en modelo físico:dispositivo simulador de lluvia (parte superior);canaleta y recipiente receptor (parte inferior).

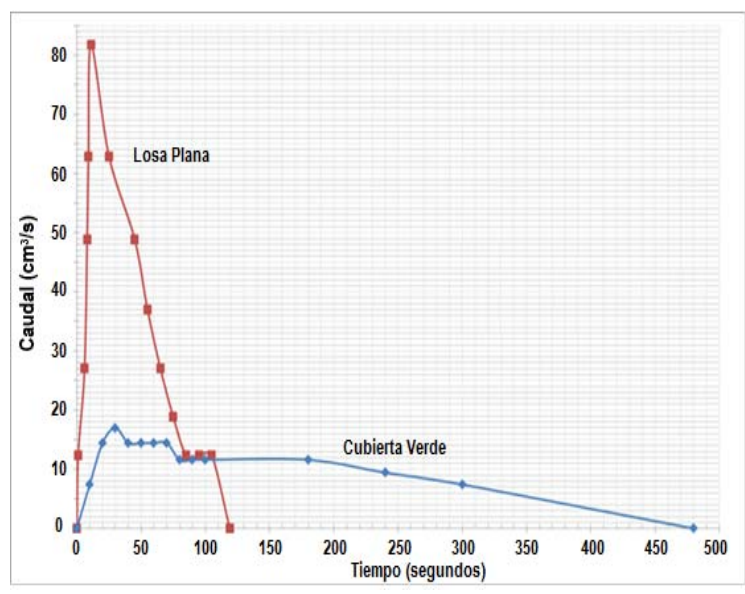

Figura 6. Comparación de hidrogramas de escurrimiento directo de los dos tipos de cubierta ensayados.

Se observó en el primer ensayo realizado sobre la cubierta ecológica una diferencia entre el volumen drenado y el precipitado de 1.1 litros, equivalente a una capacidad de retención de 8.94 litros por metro cuadrado. Esta diferencia podría explicarse por efectos físico-químicos de retención y/o evapotranspitración de la cubierta vegetal.

En los ensayos siguientes no se registraron diferencias, debido a que los sustratos del modelo se encontraban en condición de saturación.

\section{CONCLUSIONES}

De acuerdo con los ensayos realizados hasta el momento, se podría afirmar que las cubiertas verdes son eficaces, habiendo mostrado incrementos significativos de los tiempos de base y al pico del hidrograma, o sea un importante retardo el escurrimiento de las aguas de lluviay, por lo tanto, su aplicación sería beneficiosa para morigerar los problemas relacionados con la creciente impermeabilización de las ciudades que integran el AMGR.

Sin embargo, se considera que los resultados se podrían refinar cuantitativamente, debido a que no se tuvo en cuenta la reducción de la porosidad del sustrato a causa de la precipitación y las condiciones de borde del modelo, el cual no representa fielmente la realidad.

Se realizó una continuidad del presente trabajo incorporando un simulador de lluvia y la utilización de otros tipos de variedades vegetales y diferentes pendientes de cubierta. Los resultados se están procesando.

\section{REFERENCIAS BIBLIOGRÁFICAS}

Britto Correa, C. (2001). Análisis de la viabilidad y comportamiento energético de la cubierta plana ecológica. Tesis Doctoral. Universidad Politécnica de Madrid. Escuela Técnica Superior de Arquitectura de Madrid, España.

Chandías, M. E. y Ramos, J. M. (2009). Introducción a la construcción de edificios. Editorial Alsina. Buenos Aires, República Argentina. 320 pág.

Depettris, C. A. y Pilar, J.V. (2001). Uso de Medidas No Estructurales para controlar el Aumento de las Áreas Impermeables en la Ciudad de Resistencia. In I Seminario de Drenagem Urbano Do MERCOSUR - V Seminario Nacional de Drenaje Urbano: Soluções para a Drenagem Urbana em países da América Latina. Porto Alegre, RS, Brasil. 
Legislatura de la Ciudad Autónoma de Buenos Aires. (2012). Ley 4428. Disponible en http://www2.cedom.gob.ar/es/legislacion/normas/l eyes/ley4428.html.

Machado, M., Brito, C. yNeila, J. (2000). La cubierta ecológica como material de construcción.Informes de la Construcción. 52(467), 15-29.

Pilar, C.A., Cáceres, M. yBoscarino, L. (2009). Estudio de las condicionantes tecnológicas, económicas y culturales para el diseño de techos verdes. In: Comunicaciones Científicas y Tecnológicas Anuales 2009. FAU UNNE. Página 47 a 50. Corrientes. ISSN 1666-4035.

Scornik, C. (1998). Diagnóstico urbano expeditivo del A.M.G.R. SUPCE, Chaco. Informe de consultoría.

\section{Tipo de Publicación: ARTÍCULO.}

Trabajo recibido el 24/09/2019 y aprobado para su publicación el 05/11/2019.

\section{COMO CITAR}

Holsbach, N., Pilar, J. y Pilar, C. (2019). Estudio experimental del efecto hidrológico de cubiertas verdes para su utilización en el área metropolitana del Gran Resistencia. Cuadernos del CURIHAM. 25, 59-64. DOI: https://doi.org/10.35305/curiham.v25i0.121

Este es un artículo de acceso abierto bajo licencia: Creative Commons Atribución - No Comercial Compartir Igual 4.0 Internacional (CC BY-NC-SA 4.0) (https://creativecommons.org/licenses/by-ncsa/4.0/deed.es) 